\title{
Study on Paths of Affect Factors on Adaption to University Life of Chinese International Students in Korea
}

\author{
—Choosing Korean Training Chinese Students as Main Investigation Objects
}

\author{
Qinlan Lin, Ling Gao \\ College of Nursing, Beihua Uinversity, Jilin City, China \\ Email: bhdxfsyy@live.cn
}

Received April 2013

\begin{abstract}
The purpose of this paper is to study effect factors of adaptation to university life of Chinese International Students in Korea. 374 Korean language trainees are chosen as investigation objects by means of random cluster sampling. Questionnaire data are collected by using the survey form and then paths of effect factors on adaption to university life of Korean training Chinese students in Korea are analyzed. The results indicate that Korean language proficiency, physical symptoms, loneliness, self-efficacy, acculturation stress are direct effect factors on Chinese students adapting to university life in Korea. Besides, Korean language proficiency, physical symptoms, loneliness are not only direct effect factors on self-efficacy and acculturation stress but also indirect effect factors on Chinese students adapting to university life in Korea. In order to improve ability of Korean training Chinese students adapting to university life in Korea, Korean language proficiency and self-efficacy should be improved. And, some effective interventions should be given to reducing physical symptoms, loneliness and acculturation stress.
\end{abstract}

Keywords: Chinese International Students; Adaptations to University Life; Korean Language Proficiency; Loneliness; Physical Symptoms; Self-Efficacy; Acculturation Stress

\section{Introduction}

\subsection{Study Necessity}

According to statistics, $74 \%$ out of 90,658 foreign students in Korea are Chinese students [1]. It is difficult for the students in the new environment to adapt to [2] university life in Korea, as a result, some students who could not adapt to university life end up with not be able to complete their studies, consequently, they have to return home in advance, or work illegally, or become illegal residents, finally their situation get very serious [3]. The results show that the students have corresponding higher ability of adaptation to university life if they have higher Korean language proficiency [2]. The students have higher stress if they have more physical symptoms [4]. The students' ability of adaptation to university life decreases more if their loneliness and acculturation stress increase more [2]. If the students' Social support [5], self-efficacy [6] and stress response ability are higher [7], the higher ability of adaptation to university life they will have [8]. Moreover, students who live in for one to two years have the lowest level of cross-cultural adaptation, especially the psychological adaptation [9]. Therefore, Korean language training Chinese students who live less than 2 years are chosen as investigation objects, and effect factors on adaption to university life of Korean training Chinese students in Korea have to be taken into account from language, physical, society, psychology and other aspects. On the basis of the theoretical framework of combination of Roy's adaptation theory and Lazarus \& Folkman's theory of stress-appraise-coping [10], the purpose of this study is to predict effect factors on adaptation to university life of Chinese international students in Korea.

\subsection{Study Objective}

The purpose is to validate effect factors on adaptation to university life in Korea through analyzing status characteristics of Korean training Chinese students. And further, reliable basis can be provided for carrying out effective measures which is for Chinese international students better and faster adapting to university life in Korea.

\section{Research Method}

\subsection{Investigation Objective}

From June 15 to July 16, 2011, 380 Chinese international students getting undergraduate degree from the 4-year 
universities in Korea are chosen as investigation objects by means of random cluster sampling. Take back rate is $100 \%$, valid questionnaires are 374 , and response rate is $98.4 \%$. According to required sample size in complex structures model, sample size must be more than 200. In the ideal case, free parameter is 20:1. In fact, 10:1 can be used for free parameter [11]. Therefore, the sample size in this study is appropriate.

\subsection{Research Tool}

The Korean ability of self-evaluation scale made by Zhangyan (2005) is used [12] for analyzing Korean language proficiency. Dimensions and validity are investigated through pretest. There are totally 4 questions items. Points from 1 to 5 are distributed to options from "very bad" to "very good" in each item respectively. Korean language proficiency is higher if the point is higher. Cronbach's $\alpha$ is 0.86 when the scale is made, however, 0.93 is used as Cronbach's $\alpha$ in this study.

The physical symptoms scale made by Park Ji Hyun (2008) is used for analyzing physical symptoms [4]. Dimensions and validity are investigated through pretest. There are totally 18 questions items. Points from 1 to 5 are distributed to options from options from "Absolutely not" to "Often" in each item. The point is higher and physical symptoms appear more. Cronbach's $\alpha$ is 0.93 when the scale is made, however, 0.93 is used as Cronbach's $\alpha$ in this study.

The Chenjie's (2010) loneliness scale is adopted for analyzing loneliness [2]. Dimensions and validity are investigated through pretest. There are totally 20 questions items. Points from 1 to 5 are distributed to options from options from "Absolutely not" to "Absolutely" in each item. The point is higher and loneliness becomes stronger. Cronbach's $\alpha$ used by Chenjie is 0.86 , however, 0.93 is used as Cronbach's $\alpha$ in this study.

The Kim Yoon Kyoung's social support scale is used for analyzing social support [5]. Dimensions and validity are investigated through pretest. There are totally 25 questions items. Points from 1 to 5 are distributed to options from "Absolutely not" to "Absolutely" in each item. The point is higher and loneliness becomes stronger. Cronbach's $\alpha$ used by Kim Yoon Kyoung is 0.95, however, 0.96 is used as Cronbach's $\alpha$ in this study.

The Jin Gyeol's (2010) acculturation stress scale is used for analyzing acculturation stress [2]. Dimensions and validity are investigated through pretest. There are totally 28 questions items. Points from 1 to 5 are distributed to options from "Totally not" to "Totally" in each item, respectively. It indicates that acculturation stress is higher if the point is higher. Cronbach's $\alpha$ is 0.93 when the scale is made, however, 0.93 is used as Cronbach's $\alpha$ in this study.
The Lizhishan's (2010) self-efficacy scale is used to analyze self-efficacy. Dimensions and validity are investigated through pretest [8]. 5 inefficient degree items are removed, and 18 questions items are remained. Points from 1 to 5 are distributed to options from "Totally not" to "Totally" in each item, respectively. Self-efficacy is higher if the point is higher. Cronbach's $\alpha$ is 0.93 when the scale is made, also 0.93 is used as Cronbach's $\alpha$ in this study.

The Seo Ji Yeoun's (2009) stress Countermeasure scale is used to analyze stress Countermeasure [7]. Dimensions and validity are investigated through pretest. 4 inefficient degree items are removed, 10 positive questions items and 10 negative questions items are remained. Points from 1 to 4 are distributed to options from "Totally not" to "Totally" in each item, respectively. Positive and negative stresses are higher if the point is higher. Cronbach's $\alpha$ is 0.81 when the scale is made, positive Cronbach's $\alpha$ is 0.82 and negative Cronbach's $\alpha$ is 0.81 in this study.

The Zhang Yanan's (2009) adaptation to university life scale is used to analyze adaptation to university life [8]. Dimensions and validity are investigated through pretest, which includes academic adaptation, social adaptation, and emotional adaptation. There are totally 62 questions items about University environment includes. Points from 1 to 5 are distributed to options from "Totally not" to "Totally" in each item, respectively. Ability of adaptation to university life is higher if the point is higher. Cronbach's $\alpha$ is 0.95 when the scale is made, also 0.94 is used as Cronbach's $\alpha$ in this study.

\subsection{Data Collection and Analysis Method}

In accordance with the principle of informed consent, firstly, the purpose and necessity of the study should be told to investigation objects, investigation objects feel free to quit the study anytime. All of data are anonymous, and the data are collected after signing an agreement in writing. In this study, PASW Statistics 18.0 program and LISREL 8.7 program are used for the statistical analysis.

\section{Analysis Results}

\subsection{Status of Korean Training Chinese Students}

In this survey, $40.4 \%$ of students are male, and $59.6 \%$ of students are female. The average age is 20.2 years old. The average residence time is 7 months. Study satisfaction is $3.44( \pm 1.25)$ points. Option of whether to participate in Korean Language Proficiency Test (TOPIK) showed that students of $63.4 \%$ did not take TOPIK. Failure rate is $35.6 \%$ in the participants. 


\subsection{Points for Adaptation to University Life, Korean Language Proficiency, Physical Symptoms, Loneliness, Social Support, Acculturation Stress, Self-Efficacy and Stress Countermeasure}

Points of $2.78( \pm 0.43)$ is obtained when full points of university life is 5 . Points of $2.91( \pm 0.86)$ is obtained when full points of Korean language proficiency is 5 points. Points of $2.26( \pm 0.69)$ is obtained when full points of physical symptoms is 5 . Points of $3.78( \pm 0.54)$ is obtained when full points of loneliness is 5 . Points of $2.56( \pm 0.50)$ is obtained when full points of social support is 5. Points of $3.79( \pm 0.55)$ is obtained when full points of acculturation stress is 5. Points of $3.36( \pm 0.58)$ is obtained when full points of self-efficacy is 5 . Points of $2.89( \pm 0.33)$ is obtained when full points of positive stress is 4 . Points of $2.62( \pm 0.34)$ is obtained when full points of negative pressure responses is 4 .

\subsection{Analysis on Paths of Affect Factors on Adaption to University Life of Korean Training Chinese Students in Korea}

Parameter results are as follows: GFI is 0.91, AGFI is
0.92 , NNFI is 0.95 , NFI is 0.93 , RMR is 0.05 . Synthesizing exam results indicate that path analysis figure in this study has better fitting. Korean language proficiency, physical symptoms, loneliness, self-efficacy, acculturation stress in Table 1 can represent 78\% variables of affect factors on adaption to university life. Korean language proficiency, physical symptoms, loneliness, selfefficacy, acculturation stress are direct affect factors on Chinese students adapting to university life in Korea. Besides, Korean language proficiency, physical symptoms, loneliness are not only direct affect factors on selfefficacy and acculturation stress but also indirect affect factors on Chinese students adapting to university life in Korea, which is illustrated in Figure 1.

\section{Discussion}

After the text edit has been completed, the paper is ready for the template. Duplicate the template file by using the Save As command, and use the naming convention prescribed by your conference for the name of your paper. In this newly created file, highlight all of the contents and import your prepared text file. You are now ready to style your paper; use the scroll down window on the left

Table 1. Total effect of endogenous variable.

\begin{tabular}{|c|c|c|c|c|c|}
\hline Endogenous variable & Predictor variable & Standardized direct effect & Standardized indirect effect & Total effect & $\mathrm{SMC}^{\dagger}$ \\
\hline \multirow[t]{8}{*}{ Adaptations to university life } & Korean language proficiency & $0.19(2.98)^{* *}$ & $0.37(6.24)^{* * *}$ & $0.56(10.25)^{* * *}$ & 0.78 \\
\hline & Physical symptoms & $-0.16(-2.21)^{* *}$ & $-0.07(-1.83)^{* *}$ & $-0.23(-5.68)^{* *}$ & \\
\hline & Loneliness & $-0.18(-4.21)^{* * *}$ & $-0.09(-2.85)^{* *}$ & $-0.27(-6.09)^{* * * *}$ & \\
\hline & Social support & & $0.01(0.35)$ & $0.01(0.35)$ & \\
\hline & Acculturation stress & $-0.13(-2.15)^{*}$ & $-0.17(-3.62)^{* * *}$ & $-0.30(-5.25)^{* * * *}$ & \\
\hline & Self-efficacy & $0.44(2.41)^{*}$ & $0.08(0.62)$ & $0.52(5.46)^{* * *}$ & \\
\hline & Active coping & $0.11(0.54)$ & $-0.02(-0.21)$ & $0.09(0.56)$ & \\
\hline & Passivecoping & $-0.03(-0.22)$ & & $-0.03(-0.22)$ & \\
\hline
\end{tabular}

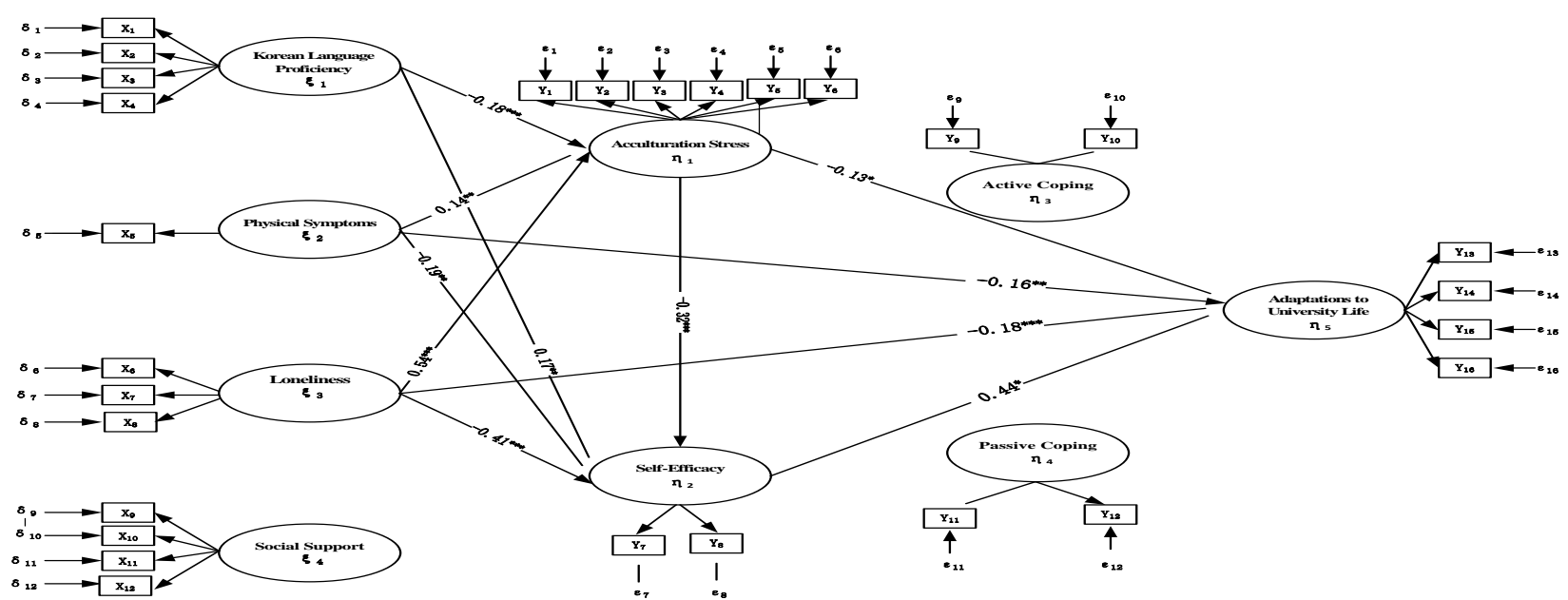

Figure 1. Paths of affect factors on adaption to university life. 
of the MS Word Formatting toolbar.

\subsection{Status of Korean Training Chinese Students}

The survey shows that students of $63.4 \%$ did not participate in TOPIK, the failure rate is $35.6 \%$, which indicates that most students did not take the test, the majority failed even though they participated in. Nowadays, TOPIK has been a necessary condition for admission and scholarship, but the realization degree on TOPIK is not enough, therefore there is an urgent need to improve realization degree on TOPIK. In addition, average points of Korean language proficiency is $2.91( \pm 0.86)$ in the results of this study, which indicates that indicating Korean language proficiency is poor. It is necessary for students to have systematic training before they study aboard. The best way is to participate in TOPIK in domestic and fully prepare. Also Korea should establish a variety of educational models for students abroad and carry out Korean training via a wide range of Korean education systems. For example, different categories of listening, speaking and writing curriculums should be established. Various activities to experience Korean culture should be organized. The assigned principle of one Korean student to one Chinese student in bedroom should be taken into account. In this way, Chinese students can chose different educations according to their need. They unknowingly learn Korean in their daily lives so that they can faster and more effectively improve their Korean language proficiency.

\subsection{Adaptation to University Life}

In this study, the average residence period of investigation objects is 7 months, the average point of adaptation to university life is $2.78( \pm 0.43)$. This result supports the point of view that students have lowest adaptation to university life [9] when they have lived for 7 - 12 months. Therefore, Korean training Chinese students whose adaptation to university life in the beginning period of study should be paid attention to. Language and environment are all strange to them in this period, various measures should be carried out to help them improve their adaptation ability so that they can successfully adapt to university life and then achieve the desired study destination.

\subsection{Affect Factors on Adaption to University Life}

This study shows that on the basis of the degreed ordering of affect factors on adaptation to university life from big to small. They are Korean language proficiency, loneliness, physical symptoms, self-efficacy, and acculturation stress, respectively. Among these factors, Korean language proficiency, loneliness, physical symptoms directly affect adaptation to university life. In addition, self-efficacy and acculturation stress have indirect effect on adaptation to university life. This result supports the point of view that Korean language proficiency is higher and adaptation to university life is higher [4], loneliness is stronger and stress is higher[2], physical symptoms appear more frequently and stress is higher, and then, self-efficacy decrease more, adaptation ability become lower [6].

When students move from a familiar environment to another unfamiliar environment, firstly, they have to adapt the language before adapting to university life. Secondly, they have to accomplish psychology and physical adaptation. Accordingly, Korean language, physical symptoms, loneliness and other direct stress sources should be paid attention to, also stress sources understood and evaluated by self-efficacy and other factors should be paid attention to. Different Korean education modes should be used. Psychological consulting rooms should be created, regular physical examination should be conducted to reduce acculturation stress and enhance self-efficacy, and these interventions above-mentioned can efficiently improve adaptation ability to university life.

This study shows that five factors explain 78\% variables of affect factors on adaption to university life, this value is higher than $48 \%$ in [2]. It indicates that the used theoretical framework of combination of Roy's adaptation theory and Lazarus \& Folkman's theory of stressappraise-coping in this study is valid. Not only is advantages of a Roy theoretical and Lazarus \& Folkman theoretical exerted, but also disadvantages of Roy theory is eked out. Affect factors on adaption to university life of Korean training Chinese students in Korea are analyzed from many different points of view.

This study shows that social support and stress interventions do not affect adaption to university Life [6], which is different from the point of view that social support is higher [6] and taken positive stress interventions to adaption is higher, however, taken negative stress interventions to adaption is lower [8]. It is recommended that further study is supposed to be researched.

\section{Conclusion}

Although effect factors on adaption to university life are complicated, they have certain logic. When interventions for improving adaptation of Korea training Chinese students in Korean are carried out, stressors namely Korean language proficiency, physical symptoms, loneliness, etc. should be paid attention to. Also, their understanding and evaluation, etc. of stressors should be regarded, which is awareness level of unfamiliar environment. Through the 
correctly evaluating stress, reducing acculturation stress and establishing a self-efficacy and other measures, the adaptation level to university life can be improved.

\section{REFERENCES}

[1] Immigration Policy Division, Korea Immigration Service, Ministry of justice. Republic of Korea, 2011. Statistics Monthly Report, Immigration Policy Division, 2011. http://www.moj.go.kr/HP/COM/bbs_03/ListShowData.do ?strNbodCd=noti0097\&strWrtNo=72\&strAnsNo=A\&str NbodCd=noti0703\&strFilePath=moj/\&strRtnURL=MOJ 40402000\&strOrgGbnCd $=104000 \&$ strThisPage $=1 \&$ strN bodCdGbn

[2] J. Chen, "The Influence of Loneliness and Acculturative Stress on Chinese Students' Adjustment to University Life,” Daegu University, Korea, 2010.

[3] H. X. Zhang, "A Study on Relationship between Social Support and Cultural Accommodation Stress among the Chinese Students in Seoul,” Department of Social Welfare, Seoul National University, Korea, 2005.

[4] J. H. Park, "The Study of Stress on Clinical Practice, Somatic Symptom, and Self-Disclosure of Nursing Students,” Catholic University of Daegu, Korea, 2008.

[5] K. Yun-Kyoung, "A Study on the Influence Factors of College Student's Adjustment," Graduate School of Public Administration, Chungnam National University, Korea.
[6] J. S. Lee, "The Influence of Self-Efficacy on Adaptation to University Student's Campus Life,” Major in Counseling Education the Graduate School of Education Sungkyunkwan University, Korea, 2009.

[7] J. Y. Seo, "The Relationship between the Way of Stress Coping and Cultural Accommodation Stress the Chinese Student,” Woosuk University, Korea, 2009.

[8] Y. N. Jiang, "Relationship between Perceived University Environment and Adjustment of University Life by Chinese Students in Korea,” Wonkwang University, Korea, 2009.

[9] H. Yuan and J. Y. Li, "Study on the Cross-Cultural Adaptation of Korean Students in Beijing," Youth Research, Vol. 2, 2009, pp. 84-93.

[10] I. Kim and M. J. Suh, "The Prediction Model of Adaptation in Patients with Rheumatoid Arthritis: Propositional Synthesis of Roys and Lazarus \& Folkmans Theory,” The Journal of Rhumatology Health, Vol. 4, No. 2, 1997, pp. $197-220$.

[11] Kline and B. Rex, "Principles and Practice of Structural Equation Modeling,” 2nd Edition, 2010.

[12] Y. Zhang, "A Study on the Korean Mastery Level and Acculturative Stress of the Chinese Students Studying in Korea,” Mokpo National University, Korea, 2005. 